Artículo

\title{
Resiliencia e implicación escolar en función del sexo y del nivel educativo en educación secundaria
}

\author{
Arantzazu Rodríguez-Fernández*, Estibaliz Ramos-Díaz, Iker Ros, Arantza Fernández-Zabala \\ y Lorena Revuelta
}

Escuela Universitaria de Magisterio, Universidad del País Vasco (Euskal Herriko Unibertsitatea), Vitoria-Gasteiz, España

\section{INFORMACIÓN DEL ARTÍCULO}

\section{Historia del artículo:}

Recibido el 9 de junio de 2015

Aceptado el 30 de septiembre de 2015

On-line el 22 de diciembre de 2015

\section{Palabras clave:}

Resiliencia

Implicación escolar

Estudiantes de educación secundaria

Psicología positiva

\begin{abstract}
R E S U M E N
El objetivo del presente estudio fue analizar las relaciones entre la resiliencia y los componentes conductual, emocional y cognitivo de la implicación escolar. Participaron 1.250 estudiantes de Educación Secundaria Obligatoria $(M=13,72, D T=1,09)$, de los cuales $612(49 \%)$ eran chicos y $638(51 \%)$ chicas. Se administraron los siguientes instrumentos de evaluación: la escala Connor-Davidson de Resiliencia (CD-RISC) y el Cuestionario de Implicación Escolar (SEM). Los resultados indican que los adolescentes resilientes presentan puntuaciones significativamente más altas que sus iguales no resilientes en implicación conductual, implicación emocional e implicación cognitiva. Este patrón de resultados es el mismo tanto para la muestra total como para las muestras en función del sexo y el nivel educativo. La discusión se centra en la importancia de fomentar el ajuste escolar de los adolescentes a través de la mejora de competencias socioemocionales.

(C) 2015 Instituto de Ciencias de la Educación de la Universidad de Oviedo. Publicado por Elsevier España, S.L.U. Este es un artículo Open Access bajo la CC BY-NC-ND licencia (http://creativecommons. org/licencias/by-nc-nd/4.0/).

\section{Resilience and school engagement by gender and education level in secondary education}

\section{A B S T R A C T}

The aim of this study was to analyse the relationships between resilience and behavioural, emotional and cognitive components of school engagement. The study included 1,250 secondary education students with a mean age of 13.72 years $(S D=1.09)$ of whom $612(49 \%)$ were male and $638(50 \%)$ female. Two assessment tools were administered: Connor-Davidson Resilience Scale (CD-RISC) and School Engagement Measurement (SEM). The results indicated that resilient adolescents showed significantly higher scores in behavioural, emotional, and cognitive engagement than their non-resilient peers. This pattern of results was the same for the total sample and for sex and education level groups. The discussion focuses on the importance of promoting school adjustment of adolescents through improvement of socio-emotional skills.

(c) 2015 Instituto de Ciencias de la Educación de la Universidad de Oviedo. Published by Elsevier España, S.L.U. This is an open access article under the CC BY-NC-ND license (http://creativecommons. org/licenses/by-nc-nd/4.0/).

\footnotetext{
* Autor para correspondencia: Departamento de Psicología Evolutiva y de la Educación, Universidad del País Vasco. E.U. Magisterio de Vitoria-Gasteiz, c/ Juan Ibañez de Sto. Domingo 1, 01006 Vitoria-Gasteiz (Álava). Tel.: +34 945013277/945014219.

Correo electrónico: arantzazu.rodriguez@ehu.eus (A. Rodríguez-Fernández).
}

\section{Introducción}

Tradicionalmente, los campos de la psicología y la educación se han orientado desde un modelo de riesgo centrado en el diseño de intervenciones preventivas en lugar de promocionar objetivos de desarrollo socioafectivo (Oliva et al., 2010), adquiriendo un protagonismo central la búsqueda de factores que influyen negativamente en la adaptación escolar del adolescente. Sin embargo, en 
las últimas décadas, es evidente el creciente interés que suscita el desarrollo de una psicología escolar positiva cuya tendencia central es el estudio de constructos psicológicos positivos (Froh, Huebner, Youssef y Conte, 2011). Desde esta perspectiva, se destacan aquellos recursos positivos o fortalezas del alumnado que permiten adquirir un funcionamiento óptimo en el entorno educativo (Kristjánsson, 2012).

El constructo de resiliencia sobresale como elemento implicado en la adaptación exitosa del ser humano (Masten y Tellegen, 2012). Es un concepto científico emergente que ha ido adquiriendo una considerable atención en el ámbito de la psicología eductiva debido al relevante rol de la escuela como promotora de bienestar (Toland y Carrigan, 2011). Ante la falta de consenso en la definición del término resiliencia (Fletcher y Sarkar, 2013), existe un acuerdo generalizado en que se trata de un proceso dinámico de naturaleza evolutiva que abarca la adaptación positiva dentro del contexto de adversidad significativa (Masten, 2014). Se puede decir que la resiliencia es un fenómeno que se caracteriza por buenos resultados a pesar de las amenazas graves para la adaptación o el desarrollo (Rutter, 2012). En este estudio la resiliencia es considerada como un conjunto de cualidades personales positivas que facilitan la adaptación individual (Connor y Davidson, 2003). En el periodo de la adolescencia, la resiliencia implica superar los efectos negativos producidos por la exposición al riesgo mediante estrategias positivas de fortalecimiento y afrontamiento (Fergus y Zimmerman, 2005). Durante esta etapa de desarrollo, los adolescentes deberán desplegar una serie de comportamientos resilientes para hacer frente a diversos desafíos y tareas evolutivas dentro del entorno escolar, tales como el incremento de las expectativas académicas y el aprendizaje de comportamientos sociales (Mahatmya, Lohman, Matjasko y Farb, 2012). El valor del enfoque de la resiliencia en el sistema educativo está asociado precisamente a la construcción de factores protectores que preparan a los estudiantes para una vida adulta sana y productiva a pesar de los factores de riesgo (Esquivel, Doll y Oades-Sese, 2011).

Una psicología escolar interesada en facilitar el funcionamiento óptimo de los estudiantes requiere la identificación de indicadores de adaptación dentro del contexto educativo, entre los que figura la implicación escolar (Veiga, García, Reeve, Wentzel y García, 2015). Este constructo se vislumbra como un factor relevante para el desarrollo sociopersonal y el éxito académico (Ros, Goikoetxea, Gairín y Lekue, 2012), y posee un significado fundamental para la comprensión del desarrollo positivo en la adolescencia (Li y Lerner, 2011). La conceptualización de la implicación escolar continúa siendo en la actualidad un debate abierto (Reschly y Christenson, 2012). Simons-Morton y Chen (2009) definen este constructo como el grado en que el alumnado se compromete con la escuela y está motivado para aprender. Actualmente existe acuerdo en considerar la implicación escolar como un meta-constructo que incluye las dimensiones conductual, emocional y cognitiva (Fredricks, Blumenfeld y Paris, 2004; Veiga, Burden, Appleton, Céu Taveira y Galvao, 2014; González y Verónica-Paoloni, 2015). Por tanto, en el presente trabajo se conceptualiza la implicación escolar como una variable multidimensional que refleja cada uno de los subtipos propuestos por Fredricks et al. (2004): participación en la escuela (implicación conductual), identificación con la escuela (implicación emocional) e inversión en la tarea de aprendizaje (implicación cognitiva).

Existe un número considerable de estudios que apoyan la asociación entre determinadas variables psicológicas como la autoeficacia y el autoconcepto con la implicación escolar (Veiga et al., 2014). A pesar de que los antecedentes de investigación que se aproximan al análisis de la relación entre la resiliencia y la implicación escolar son escasos, cabe figurarse una correlación positiva entre ambas variables, de manera que los estudiantes que responden de manera resiliente ante los estresores del contexto escolar, despliegan reacciones adaptativas hacia el centro educativo y lo académico, participan en la escuela y dedican más tiempo a las tareas de aprendizaje que los no resilientes. Entre los estudios que consideran la resiliencia como una característica individual que pronostica la adaptación dentro del entorno escolar, destaca la investigación de Sharkey, You y Schnoebelen (2008), que revela el rol mediador de la resiliencia (compuesta por factores como la cooperación, empatía, resolución de problemas o autoeficacia entre otros) entre los recursos escolares y la implicación emocional en una muestra de 20.000 estudiantes adolescentes. También se encuentra que la mejora de la implicación escolar puede derivar de los esfuerzos escolares destinados a mejorar aspectos resilientes en una muestra de jóvenes estudiantes (Ungar y Liebenberg, 2013). En otra publicación reciente, Jones y Lafreniere (2014) señalan una significativa relación entre resiliencia e implicación escolar en estudiantes expuestos a situaciones de pobreza. No obstante, ninguno de los estudios referenciados analizan la implicación escolar desde una perspectiva multidimensional ni incorporan un análisis de esta relación teniendo en cuenta el sexo y el nivel educativo. En una línea de investigación diferente, también se encuentran estudios en los que la resiliencia es considerada como variable resultante de la implicación escolar de adolescentes afroamericanos de bajos ingresos y de zonas rurales (Irvin, 2012) y menores en situación de desprotección grave (Malindi y Machenjedze, 2012).

La investigación científica sobre las diferencias según el sexo y el nivel educativo en las variables estudiadas ha sido limitada; por un lado, las escasas investigaciones que han abordado las diferencias de sexo y edad en resiliencia sugieren diferencias significativas a favor del sexo masculino (Deb y Arora, 2009; González-Arratia, Valdez y Zavala, 2008) y la disminución del nivel de resiliencia durante la etapa de la adolescencia (Masten, 2007). Por otro lado, los estudios que abordan las diferencias de sexo y nivel educativo en la implicación escolar revelan diferencias significativas en la implicación escolar a favor de las estudiantes de sexo femenino (Lam, Wong, Yang y Liu, 2012) y un descenso de la misma durante la transición de la etapa de educación primaria a la de secundaria (Mahatmya et al., 2012). Por lo tanto, existen razones para analizar empíricamente las diferencias de sexo y nivel educativo en la resiliencia y la implicación del estudiante.

Teniendo en cuenta estos precedentes, el presente trabajo tiene como objetivo explorar las relaciones entre la resiliencia y los componentes conductual, emocional y cognitivo de la implicación escolar. Se espera que exista una conexión directa de la resiliencia con los componentes de la implicación escolar y que los adolescentes resilientes presenten puntuaciones significativamente más altas en todos los dominios de la implicación escolar. Estos resultados son esperables para la muestra total, mientras que en el caso de los análisis por sexo y nivel educativo se esperan diferencias acordes con la investigación previa señalada.

\section{Método}

\section{Participantes}

La muestra se compuso de un total de 1.250 estudiantes $(49 \%$ chicos y $51 \%$ chicas) de entre 12 y 15 años de edad $(M=13,72$, $D T=1,09$ ) pertenecientes a 9 centros educativos públicos y concertados de la Comunidad del País Vasco. Se utilizó un muestreo aleatorizado estratificado, a través de colaboradores, tratando de abarcar la representatividad de los estudiantes de secundaria de la Comunidad del País Vasco. La distribución de los participantes según el ciclo escolar fue la siguiente: 504 estudiantes de 1 . $^{\text {er }}$ ciclo de ESO (40,3\%); y 746 estudiantes de $2 .^{\circ}$ ciclo de ESO (59,7\%). La Chi cuadrado de Pearson indicó que no había diferencias en 
Tabla 1

Coeficientes de correlación de Pearson entre la resiliencia y las escalas de implicación escolar, y correlaciones parciales controlando sexo y ciclo escolar

\begin{tabular}{|c|c|c|c|c|c|c|}
\hline & $\begin{array}{l}\text { Muestra total } \\
(\mathrm{n}=1.250)\end{array}$ & $\begin{array}{l}\text { Correlaciones controlando } \\
\text { efecto sexo y ciclo escolar } \\
(\mathrm{n}=1.250)\end{array}$ & Chicos $(n=612)$ & Chicas $(n=638)$ & $\begin{array}{l}\text { 1. }{ }^{\mathrm{er}} \text { ciclo ESO } \\
(\mathrm{n}=504)\end{array}$ & $\begin{array}{l}\text { 2. }{ }^{\circ} \text { ciclo ESO } \\
(\mathrm{n}=746)\end{array}$ \\
\hline \multicolumn{7}{|l|}{ Resiliencia } \\
\hline Implicación conductual & $0,232^{* *}$ & $0,260^{* *}$ & $0,225^{* *}$ & $0,321^{* *}$ & $0,251^{* *}$ & $0,198^{* *}$ \\
\hline Implicación emocional & $0,329^{* *}$ & $0,354^{* *}$ & $0,309^{* *}$ & $0,426^{* *}$ & $0,310^{* *}$ & $0,321^{* *}$ \\
\hline Implicación cognitiva & $0,309^{* *}$ & $0,300^{* *}$ & $0,301^{* *}$ & $0,330^{* *}$ & $0,297^{* *}$ & $0,295^{* *}$ \\
\hline
\end{tabular}

${ }^{* *} p<0,01$.

la distribución del número de participantes de cada sexo en los diferentes niveles educativos $\left(\chi^{2}{ }_{(1)}=4,66, p>0,05\right)$.

\section{Instrumentos}

Escala de resiliencia Connor-Davidson (CD-RISC; Connor y Davidson, 2003). Es un cuestionario autoaplicado de 25 ítems (ejemplos: soy capaz de adaptarme a los cambios; me esfuerzo en conseguir mis objetivos) que se responden en una escala tipo Likert que oscila desde $0=$ nada de acuerdo a 4 =totalmente de acuerdo. El instrumento ha demostrado tener una adecuada consistencia interna, fiabilidad test-retest, validez convergente y divergente (Connor y Davidson, 2003). El análisis factorial desarrollado por los autores sugiere una estructura de cinco factores: competencia personal, confianza en la intuición y tolerancia a la adversidad, aceptación positiva al cambio, control y espiritualidad. Algunos estudios señalan la inestabilidad del modelo pentadimensional y la necesidad de afianzar su estructura (Prince-Embury, 2013). Al igual que otros autores (Campbell-Sills, Cohan y Stein, 2006) en los análisis se utilizó la puntuación total de los ítems, con una consistencia interna (alpha de Cronbach) elevada $(\alpha=0,86)$.

Cuestionario de implicación escolar (SEM) de Fredricks, Blumenfeld, Friedel y Paris (2005). Está compuesto por 19 ítems (ejemplos: me divierto estando en clase; presto atención en clase) con un formato de respuesta Likert de 5 grados (donde $1=$ nunca y $5=$ siempre). El análisis factorial de los elementos da lugar a tres subescalas: implicación conductual, implicación emocional e implicación cognitiva. Los autores informan de múltiples análisis que confirman la validez del cuestionario (Blumenfeld et al., 2005; Fredricks et al., 2005; Fredricks y McColskey, 2012). Las propiedades psicométricas del instrumento aportadas por la muestra del presente estudio indican índices de consistencia interna de $\alpha=0,81$ para la escala de implicación emocional, de $\alpha=0,74$ para la implicación conductual y de $\alpha=0,77$ para la implicación cognitiva.

\section{Procedimiento}

En un primer momento se contactó con la dirección de los centros educativos explicando el proyecto de investigación. Una vez confirmada su participación, se entregaron los consentimientos informados para los padres y los escolares adolescentes. Posteriormente, dos investigadoras entrenadas en la aplicación de los cuestionarios acudieron a los centros y administraron a los participantes los instrumentos de evaluación dentro del horario de clase y de manera simultánea a todo el alumnado integrante de un mismo aula. Los datos se recogieron durante los meses de octubre y marzo del curso académico 2012-2013. Con el propósito de mitigar respuestas en dirección de las hipótesis de la investigación, se empleó el criterio de ciego único, evitando que los estudiantes conocieran la finalidad del estudio. La confidencialidad y voluntariedad fueron asimismo garantizadas para reducir los efectos de la deseabilidad social.

\section{Análisis de datos}

En primer lugar, se llevó a cabo un análisis de las correlaciones entre la resiliencia y las dimensiones de la implicación escolar. En segundo lugar, se agrupó la muestra en tres perfiles en función de las puntuaciones obtenidas en la CD-RISC: resiliencia baja (percentil<20), resiliencia media (percentil 20-80) y resiliencia alta (percentil> 80). Así, en la muestra del estudio los valores extremos (resiliencia baja y resiliencia alta) del CD-RISC suponen un $10 \%$, dejando un rango del $80 \%$ de los casos para la resiliencia de nivel moderado. Finalmente, se desarrollaron los siguientes análisis: descriptivos (medias y desviaciones típicas); de varianza en función del nivel de resiliencia (ANOVA) por separado para la muestra total y para cada una de las submuestras; del tamaño del efecto (Eta); y post hoc de comparaciones múltiples (Bonferroni). Respecto a la estimación del tamaño del efecto, la regla de Cohen (1988) señala las siguientes estimaciones: 0,01 -0,06 (efecto pequeño); 0,06-0,14 (efecto medio); >0,14 (efecto grande).

\section{Resultados}

Correlaciones entre resiliencia y dimensiones de la implicación escolar

Los coeficientes de correlación de Pearson de la resiliencia con las dimensiones de la implicación escolar (conductual, emocional y cognitiva) calculados para el conjunto de la muestra y las submuestras (chicos, chicas, $1 .^{\text {er }}$ ciclo ESO y $2 .^{\circ}$ ciclo ESO), así como las correlaciones parciales controlando sexo y ciclo escolar, se presentan en la tabla 1.

Los datos expuestos indican que todas las correlaciones resultan ser significativas y de signo positivo $(p<0,01)$. Es decir, la resiliencia y las tres facetas de implicación escolar covarían de forma parecida, de tal modo que los adolescentes más resilientes se encuentran escolarmente implicados y viceversa. Cabe destacar que se obtiene la correlación más alta entre la resiliencia y la implicación emocio$\operatorname{nal}(r=0,329, p<0,01)$. Tanto en la muestra de sexo masculino como en la de sexo femenino los coeficientes obtenidos evidenciaron nuevamente correlaciones significativas $(p<0,01)$ entre la resiliencia y los componentes de la implicación escolar. Igualmente, relaciones directas estadísticamente significativas $(p<0,01)$ fueron encontradas en ambos grupos de ciclo educativo (1. ${ }^{\circ}$ y $2 .^{\circ}$ de ESO). Por último, las correlaciones parciales entre la resiliencia y las dimensiones de la implicación escolar controlando simultáneamente el efecto del sexo y el nivel educativo muestran resultados similares (implicación conductual: $r=0,260, p<0,01$; implicación emocional: $r=0,354, p<0,01$; implicación cognitiva: $r=0,300, p<0,01$ ), lo que significa que ambas variables sociopersonales no poseen un efecto mediador.

\section{Resiliencia e implicación conductual}

Los datos de la tabla 2 señalan que aquellos estudiantes de la muestra total que manifestaron valoraciones altas (percentil > 80) 
Tabla 2

Implicación conductual en función del nivel de resiliencia

\begin{tabular}{|c|c|c|c|c|c|}
\hline Resiliencia baja M (DT) & Resiliencia media M (DT) & Resiliencia alta M (DT) & $\mathrm{F}$ & $\eta^{2}$ & Post hoc \\
\hline \multicolumn{6}{|l|}{$\begin{array}{l}\text { Implicación conductual } \\
\text { Muestra total }(n=1.250)\end{array}$} \\
\hline $18,24(2,84)$ & $19,34(2,88)$ & $20,29(2,89)$ & $25,07^{*}$ & 0,039 & $1<2 ; 1<3 ; 2<3$ \\
\hline Chicos $(n=612)$ & & & & & \\
\hline $17,79(2,98)$ & $18,62(2,89)$ & $19,87(3,03)$ & $12,76^{*}$ & 0,040 & $1<3 ; 2<3$ \\
\hline $\begin{array}{c}\text { Chicas }(n=638) \\
18,47(2,75)\end{array}$ & $20,04(2,70)$ & $21,02(2,47)$ & $25,52^{*}$ & 0,074 & $1<2 ; 1<3$ \\
\hline $\begin{array}{l}\text { 1. }{ }^{e r} \text { ciclo ESO }(n=504) \\
18,48(2,94)\end{array}$ & $19,99(2,91)$ & $20,80(2,62)$ & $14,21^{*}$ & 0,054 & $1<2 ; 1<3 ; 2<3$ \\
\hline $\begin{array}{l}\text { 2. }{ }^{\circ} \text { ciclo } \operatorname{ESO}(n=746) \\
18,10(2,78)\end{array}$ & $18,92(2,79)$ & $19,81(3,05)$ & $10,13^{*}$ & 0,027 & $1<2 ; 1<3 ; 2<3$ \\
\hline
\end{tabular}

Resiliencia baja (percentil<20); resiliencia media (percentil 20-80); resiliencia alta (percentil> 80).

$\eta^{2}$ (eta estadística al cuadrado): estimaciones del tamaño del efecto; Post hoc: prueba de comparación de grupos de Bonferroni. ${ }^{*} p<0,05$.

Tabla 3

Implicación emocional en función del nivel de resiliencia

\begin{tabular}{|c|c|c|c|c|c|}
\hline Resiliencia baja M (DT) & Resiliencia media M (DT) & Resiliencia alta M (DT) & $\mathrm{F}$ & $\eta^{2}$ & Post hoc \\
\hline \multicolumn{6}{|l|}{ Implicación emocional } \\
\hline $19,72(4,13)$ & $21,88(3,81)$ & $23,82(4,03)$ & $55,64^{*}$ & 0,082 & $1<2 ; 1<3 ; 2<3$ \\
\hline \multicolumn{6}{|l|}{ Chicos $(n=612)$} \\
\hline $19,41(4,35)$ & $20,93(3,91)$ & $23,10(3,97)$ & $21,53^{*}$ & 0,066 & $1<2 ; 1<3 ; 2<3$ \\
\hline \multicolumn{6}{|l|}{ Chicas $(n=638)$} \\
\hline $19,87(4,03)$ & $22,81(3,46)$ & $25,08(3,12)$ & $56,68^{*}$ & 0,151 & $1<2 ; 1<3 ; 2<3$ \\
\hline $\begin{array}{l}1^{\text {er }} \text { ciclo ESO }(n=504) \\
20,51(3,82)\end{array}$ & $23,19(3,64)$ & $24,50(4,09)$ & $24,52^{*}$ & 0,089 & $1<2 ; 1<3 ; 2<3$ \\
\hline \multicolumn{6}{|l|}{ 2. ${ }^{\circ}$ ciclo ESO $(n=746)$} \\
\hline
\end{tabular}

Resiliencia baja (percentil<20); resiliencia media (percentil 20-80); resiliencia alta (percentil> 80).

$\eta^{2}$ (eta estadística al cuadrado): estimaciones del tamaño del efecto; Post hoc: prueba de comparación de grupos de Bonferroni. ${ }^{*} p<0,05$.

y moderadas (percentil 20-80) en resiliencia, en comparación con los no resilientes (percentil $<20$ ), presentaban puntuaciones significativamente más altas $(p<0,05)$ en implicación conductual. Este patrón de resultados es similar en los chicos y en las chicas, así como en ambos grupos de ciclo escolar. La magnitud del tamaño del efecto es pequeña para todos los grupos salvo para el grupo de sexo femenino, que presenta una intensidad moderada. Las diferencias en los contrastes Bonferroni fueron significativas en la mayoría de los perfiles de resiliencia, pero no para la comparación resiliencia baja versus resiliencia media en la muestra de sexo masculino ni para la comparación resiliencia media versus resiliencia alta en la muestra de sexo femenino.

\section{Resiliencia e implicación emocional}

Tal y como se puede comprobar en la tabla 3, los chicos y chicas adolescentes con un perfil alto (percentil $>80$ ) y moderado (percentil 20-80) de resiliencia comparados con los de perfil bajo (percentil $<20$ ), se encuentran significativamente más implicados a nivel emocional con el centro educativo en todas las muestras del estudio. En relación al tamaño del efecto, puede observarse que su magnitud es grande en el grupo de chicas, y moderada en el resto de los grupos. En base a las pruebas post hoc, mediante el estadístico Bonferroni, se puede comprobar que cualquier incremento en la resiliencia implica invariablemente un aumento de la implicación emocional, por lo que todas las comparaciones fueron estadísticamente significativas.

\section{Resiliencia e implicación cognitiva}

La relación entre resiliencia e implicación cognitiva (tabla 4) sigue el mismo patrón de resultados que el resto de las escalas del SEM (implicación conductual e implicación emocional). Las diferencias responden al siguiente patrón: los estudiantes que presentan mayores niveles de resiliencia son, cuando se les compara con los restantes grupos, los que informan de mayores niveles de implicación cognitiva. Los coeficientes del tamaño del efecto son de amplitud moderada para todos los grupos. Los contrastes post hoc, indican que la implicación cognitiva de los adolescentes según el perfil de resiliencia mostró diferencias estadísticamente significativas en todos los perfiles, excepto en la muestra de sexo masculino entre los perfiles de resiliencia baja y resiliencia alta.

\section{Discusión}

Más allá de la perspectiva académica, la finalidad principal del sistema educativo es fomentar el desarrollo positivo del alumnado. Un renovado interés por la adaptación de los escolares adolescentes y la responsabilidad de los centros educativos en la promoción del ajuste escolar han impactado en la investigación psicoeducativa, predominando en la actualidad una tendencia a analizar los recursos que inciden sobre la adaptación escolar (Kristjánsson, 2012). Conseguir la implicación del estudiante es uno de los retos más importantes del sistema educativo, ya que esta respuesta psicológica supone una manifestación evidente del ajuste adolescente en 
Tabla 4

Implicación cognitiva en función del nivel de resiliencia

\begin{tabular}{|c|c|c|c|c|c|}
\hline Perfil 1 M (DT) & Perfil $2 \mathrm{M}$ (DT) & Perfil 3 M (DT) & $\mathrm{F}$ & $\eta^{2}$ & Post hoc \\
\hline \multicolumn{6}{|c|}{ Implicación cognitiva } \\
\hline $20,40(5,32)$ & $22,76(5,36)$ & $25,86(6,24)$ & $48,51^{*}$ & 0,072 & $1<2 ; 1<3 ; 2<3$ \\
\hline $\begin{array}{c}\text { Chicos }(n=612) \\
20,38(5,79)\end{array}$ & $22,42(5,42)$ & $25,58(6,42)$ & $21,51^{*}$ & 0,066 & $1<3 ; 2<3$ \\
\hline $\begin{array}{c}\text { Chicas }(n=638) \\
20,41(5,09)\end{array}$ & $23,10(5,29)$ & $26,34(5,93)$ & $29,51^{*}$ & 0,085 & $1<2 ; 1<3 ; 2<3$ \\
\hline $\begin{array}{r}1 .{ }^{e r} \text { ciclo ESO (n } \\
21,31(5,13)\end{array}$ & $24,41(5,58)$ & $27,42(5,99)$ & $25,07^{*}$ & 0,091 & $1<2 ; 1<3 ; 2<3$ \\
\hline $\begin{array}{l}\text { 2. ciclo ESO (n } \\
19,85(5,38)\end{array}$ & $21,70(4,94)$ & $24,38(6,14)$ & $20,88^{*}$ & 0.053 & $1<2 ; 1<3 ; 2<3$ \\
\hline
\end{tabular}

Resiliencia baja (percentil<20); resiliencia media (percentil 20-80); resiliencia alta (percentil> 80).

$\eta^{2}$ (eta estadística al cuadrado): estimaciones del tamaño del efecto. Post hoc: prueba de comparación de grupos de Bonferroni. $p<0,05$.

la escuela (Griffiths, Sharkey y Furlong, 2009). Con base en la psicología positiva, el presente trabajo explora las relaciones entre la resiliencia y los componentes conductual, emocional y cognitivo de la implicación escolar.

Los resultados han aportado una evidencia inicial sobre el papel que cumple la resiliencia en la implicación escolar del alumnado de ESO, contribuyendo al conocimiento actual sobre dicha relación al tratarse de un área de investigación apenas explorada. Como aportación novedosa destaca el análisis de la relación resilienciaimplicación escolar desde una perspectiva tridimensional de la implicación escolar teniendo en cuenta el sexo y el nivel educativo del estudiantado. Los datos obtenidos sugieren que los alumnos resilientes presentan una mayor implicación con el centro educativo que los alumnos no resilientes. Cabe señalar que las diferencias de mayor magnitud se dieron en relación a las dimensiones de implicación emocional e implicación cognitiva, y aunque también pero en menor medida, en la implicación conductual. Además, se hallaron tamaños del efecto de magnitud moderada que apoyan la relevancia empírica de estas diferencias (Sun, Pan y Wang, 2010). Dicho de otro modo, los adolescentes resilientes se sienten más identificados con la escuela, invierten más en la tarea de aprendizaje y también, aunque en menor medida, son más participativos en la escuela que los no resilientes. Asimismo, se puede advertir que, en todas las facetas de la implicación escolar, las variaciones de mayor magnitud en función del perfil de resiliencia se asocian al sexo femenino y al $1^{\mathrm{er}}$ ciclo de ESO. Estos datos apoyan que el afrontamiento resiliente del alumnado está especialmente vinculado con la implicación escolar en el caso de las chicas adolescentes y los estudiantes que cursan $1^{\mathrm{er}}$ ciclo de ESO en comparación con los chicos adolescentes y los estudiantes que cursan $2 .^{\circ}$ ciclo de ESO. A este respecto, Lam et al. (2012) sugieren que la consideración de la implicación escolar como un factor de motivación y personalidad podría explicar las diferencias intersexo en los niveles de implicación con el centro educativo. Por otro lado, la disminución de la implicación escolar a lo largo de los años de escolaridad podría estar relacionada con los cambios en la influencia del grupo de iguales (Li, Lynch, Kalvin, Liu y Lerner, 2011).

Los resultados de este trabajo confirman los obtenidos en otra investigación previa que también ha señalado una significativa relación entre resiliencia e implicación escolar (Jones y Lafreniere, 2014), y apuntan en la misma dirección que trabajos que indican que la resiliencia es un factor influyente en la implicación escolar (Sharkey et al., 2008) o que la mejora de la implicación de los estudiantes puede derivar de los esfuerzos destinados a mejorar aspectos resilientes (Ungar y Liebenberg, 2013). La consistencia de los resultados sobre la relación positiva entre la resiliencia y la implicación escolar, pone de manifiesto la relevancia de la resiliencia como factor crucial del ajuste escolar adolescente.

Como limitación del estudio cabe destacar que, debido a su naturaleza transversal, no es posible extraer conclusiones relativas a la relación causal entre la resiliencia y las dimensiones de la implicación escolar. Futuras investigaciones podrían profundizar en la realización de un estudio de diseño longitudinal de carácter prospectivo, además del uso de análisis estadísticos como los modelos de ecuaciones estructurales. Además, es posible que otros aspectos influyan en la correlación entre ambas variables, ya que algunos autores afirman que el autoconcepto guarda una relación directa con la implicación del alumnado en el proceso de aprendizaje (Inglés, Martínez-Monteagudo, García-Fernández, Valle y Castejón, 2014) y otros van un paso más allá afirmando que la implicación escolar podría estar mediando entre el autoconcepto y el rendimiento académico (Green et al., 2012). Por tanto, los resultados obtenidos en este estudio podrían matizarse incluyendo otras variables como el autoconcepto y el rendimiento académico que también han demostrado correspondencia con la implicación del estudiante. Finalmente, se ha de tener en cuenta que los resultados de este trabajo son exploratorios y se ajustan a una muestra de estudiantes de secundaria con edades comprendidas entre los $12 \mathrm{y}$ los 15 años de edad, por lo que no son generalizables a estudiantes de otras edades o etapas educativas; en este sentido, se debería ampliar el estudio a otras edades.

Los resultados tienen implicaciones educativas y permiten sugerir la necesidad de fomentar el desarrollo psicosocial en contextos educativos (Madariaga y Goñi, 2009) a través del fomento de recursos personales como la resiliencia que ofrecen a la psicología de la educación una oportunidad de movimiento más allá del modelo del déficit en el ámbito aplicado (Tolan y Carrigan, 2011). Precisamente, es constatable el hecho de que las investigaciones sobre la aplicación de intervenciones con base empírica destinadas a potenciar la resiliencia en la escuela están cogiendo impulso (Prince-Embury y Saklofske, 2014) a medida que se confirma que el afrontamiento adecuado de la adversidad mejora los sentimientos, pensamientos y conductas manifestadas por los estudiantes acerca de sus experiencias en el contexto escolar. Del mismo modo, los resultados de este estudio enfatizan la importancia de implementar a nivel práctico programas de intervención psicoeducativa con el objeto de prevenir la falta de implicación escolar desde un modelo de fortalecimiento centrado en la resiliencia. A la luz de los hallazgos recientes (Song, Sikorski, Doll y Sikorski, 2014), los programas de intervención psicológica para fomentar la resiliencia y la implicación escolar deben promover el trabajo en equipo y el liderazgo mientras se trabajan el autoconcepto y 
el empoderamiento, así como considerar factores ambientales del aula como la colaboración entre profesorado y alumnado.

\section{Financiación}

Los autores del presente estudio forman parte del Grupo Consolidado de Investigación IT701-13 del Sistema Universitario Vasco. El estudio se llevó a cabo como parte del proyecto de investigación EHUA 13/26 en la Universidad del País Vasco.

\section{Conflicto de intereses}

Los autores declaran no tener ningún conflicto de intereses.

\section{Referencias bibliográficas}

Campbell-Sills, L., Cohan, S. L. y Stein, M. B. (2006). Relationship of resilience to personality, coping, and psychiatric symptoms in young adults. Behaviour Research and Therapy, 44(4), 585-599. http://dx.doi.org/10.1016/j.brat.2005.05.001

Cohen, J. (1988). Statistical power analysis for the behavioural sciences (2. ${ }^{\mathrm{a}}$ ed.). New York: Academic Press.

Connor, K. M. y Davidson, J. T. (2003). Development of a new resilience scale: The Connor-Davidson Resilience Scale (CD-RISC). Depression and Anxiety, 18(2), 76-82. http://dx.doi.org/10.1002/da.10113

Deb, A. y Arora, M. (2009). Gender differences in the perception of academic adversity and resilience among indian adolescents. Indian Journal of Community Psychology, 5(2), 158-175.

Esquivel, G., Doll, B. y Oades-Sese, G. (2011). Introduction to the especial issue: resilience in schools. Psychology in the Schools, 48(7), 649-651. http://dx.doi.org/ 10.1002/pits.20585

Fergus, S. y Zimmerman, M. A. (2005). Adolescent resilience: A framework for understanding healthy development in the face of risk. Annual Review of Public Health, 26, 399-419. http://dx.doi.org/10.1146/annurev.pubhealth. 26.021304.144357

Fletcher, D. y Sarkar, M. (2013). Psychological resilience: A review and critique of definitions, concepts, and theory. European Psychologist, 18(1), 12-23. http://dx.doi.org/10.1027/1016-9040/a000124

Fredricks, J. A., Blumenfeld, P. C. y Paris, A. H. (2004). School engagement: potential of the concept, state of the evidence. Review of Educational Research, 74(1), 59-109. http://dx.doi.org/10.3102/00346543074001059

Fredricks, J. A., Blumenfeld, P. C., Friedel, J. y Paris, A. (2005). School engagement. En K. A. Moore y L. Lippman (Eds.), Conceptualizing and measuring indicators of positive development: What do children need to flourish (pp. 305-321). New York: Kluwer Academic/Plenum Press.

Fredricks, J. A. y McColskey, W. (2012). The measurement of student engagement: A comparative analysis of various methods and student self-report instruments. En S. L. Christenson, A. L. Reschly, C. Wylie, S. L. Christenson, A. L. Reschly, y C. Wylie (Eds.), Handbook of research on student engagement (pp. 763-782). New York, NY, US: Springer. http://dx.doi.org/10.1007/978-1-4614-2018-7_37

Froh, J.J., Huebner, E., Youssef, A. y Conte, V. (2011). Acknowledging and appreciating the full spectrum of the human condition: School psychology's (limited) focus on positive psychological functioning. Psychology in the Schools, 48(2), 110-123. http://dx.doi.org/10.1002/pits.20530

González-Arratia, N., Valdez, J. y Zavala, Y. (2008). Resiliencia en adolescentes mexicanos. Enseñanza e investigación en Psicología, 13(1), 41-52.

González, A. y Verónica-Paoloni, P. (2015). Implicacioń y rendimiento en Fiśica: el papel de las estrategias docentes en el aula, y el interés personal y situacional. Revista de Psicodidáctica, 20(1) http://dx.doi.org/10.1387/RevPsicodidact.11370

Green, J., Liem, G. D., Martin, A. J., Colmar, S., Marsh, H. W. y McInerney, D. (2012). Academic motivation, self-concept, engagement, and performance in high school: Key processes from a longitudinal perspective. Journal of Adolescence, 35(5), 1111-1122. http://dx.doi.org/10.1016/j.adolescence.2012.02.016

Griffiths, A., Sharkey, J. D. y Furlong, M. J. (2009). Student engagement and positive school adaptation. En R. Gilman, E. Huebner, y M. J. Furlong (Eds.), Handbook of positive psychology in schools (pp. 197-211). New York, NY, US: Routledge/Taylor \& Francis Group.

Inglés, C., Martínez-Monteagudo, M., García-Fernández, J., Valle, A. y Castejón, J. (2014). Perfiles de orientaciones de metas y autoconcepto de estudiantes de Educación Secundaria. Revista de Psicodidáctica, 20(1) http://dx.doi.org/ 10.1387/RevPsicodidact.1023

Irvin, M. J. (2012). Role of student engagement in the resilience of African American adolescents from low-income rural communities. Psychology in the Schools, 49(2), 176-193. http://dx.doi.org/10.1002/pits.20626

Jones, G. y Lafreniere, K. (2014). Exploring the role of school engagement in predicting resilience among bahamian youth. Journal of Black Psychology, 40(1), 47-68. http://dx.doi.org/10.1177/0095798412469230

Kristjánsson, K. (2012). Positive psychology and positive education: Old wine in new bottles? Educational Psychologist, 47(2), 86-105. http://dx.doi.org/ 10.10080/00461520.2011.610678
Lam, S., Wong, B. H., Yang, H. y Liu, Y. (2012). Understanding student engagement with a contextual model. En S. L. Christenson, A. L. Reschly, y C. Wylie (Eds.), Handbook of research on student engagement (pp. 403-419). New York, NY US: Springer Science. http://dx.doi.org/10.1007/978-1-4614-2018-7_19

Li, Y. y Lerner, R. M. (2011). Trajectories of school engagement during adolescence: Implications for grades, depression, delinquency, and substance use. Developmental Psychology, 47(1), 233-247. http://dx.doi.org/10.1037/a0021307

Li, Y., Lynch, A. D., Kalvin, C., Liu, J. y Lerner, R. M. (2011). Peer relationships as a context for the development of school engagement during early adolescence. International Journal of Behavioral Development, 35(4), 329-342. http://dx.doi.org/10.1177/0165025411402578

Madariaga, J. M. y Goñi, A. (2009). El desarrollo psicosocial. Revista de Psicodidáctica, 14(1), 93-118.

Mahatmya, D., Lohman, B. J., Matjasko, J. L. y Farb, A. (2012). Engagement across developmental periods. En S. L. Christenson, A. L. Reschly, y C. Wylie (Eds.), Handbook of research on student engagement (pp. 45-63). New York, NY US: Springer Science+Business Media. http://dx.doi.org/10.1007/978-1-4614-2018-7_3

Malindi, M. y Machenjedze, N. (2012). The role of school engagement in strengthening resilience among male street children. South African Journal of Psychology, 42(1), 71-81.

Masten, A. S. (2007). Competence, resilience, and development in adolescence: Clues for prevention science. En D. Romer y E. F. Walker (Eds.), Adolescent psychopathology and the developing brain: Integrating brain prevention science ( $\mathrm{pp}$. 31-52). New York, NY US: Oxford University Press. http://dx.doi.org/10.1093 acprof:oso/9780195306255.003.0002

Masten, A. S. (2014). Invited commentary: Resilience and positive youth development frameworks in developmental science. Journal of Youth and Adolescence, 43(6), 1018-1024. http://dx.doi.org/10.1007/s10964-014-0118-7

Masten, A. S. y Tellegen, A. (2012). Resilience in developmental psychopathology: Contributions of the Project Competence Longitudinal Study Development and Psychopathology, 24(2), 345-361. http://dx.doi.org/10.1017/ S095457941200003X

Oliva, A., Ríos, M., Antolín, L., Parra, Á., Hernando, Á. y Pertegal, M. (2010). Más allá del déficit: construyendo un modelo de desarrollo positivo adolescente. Infancia y Aprendizaje, 33(2), 223-234. http://dx.doi.org/10.1174/021037010791114562

Prince-Embury, S. (2013). The Connor-Davidson Resilience Scale. En S. PrinceEmbury y D. H. Saklofske (Eds.), Resilience in children, adolescents, and adults: Translating research into practice (pp. 161-166). New York, NY US: Springe Science. http://dx.doi.org/10.1007/978-1-4614-4939-312

Prince-Embury, S. y Saklofske, D. H. (2014). Resilience interventions for youth in diverse populations. New York, NY, US: Springer Science+Business Media. http://dx.doi.org/10.1007/978-1-4939-0542-3

Reschly, A. L. y Christenson, S. L. (2012). Jingle, jangle, and conceptual haziness: Evolution and future directions of the engagement construct. En S. L. Christenson, A. L. Reschly, y C. Wylie (Eds.), Handbook of research on student engagement (pp. 3-19). New York, NY, US: Springer Science. http://dx.doi.org/ 10.1007/978-1-4614-2018-7_1

Ros, I., Goikoetxea, J., Gairín, J. y Lekue, P. (2012). Implicación del alumnado en la escuela: diferencias interindividuales e intercentros. Revista de Psicodidáctica, 17(2), 291-307. http://dx.doi.org/10.1387/Rev.Psicodidact.4557

Rutter, M. (2012). Resilience: Causal pathways and social ecology. En M. Ungar (Ed.), The social ecology of resilience: $A$ handbook of theory and practice (pp. 33-42). New York, NY, US: Springer Science. http://dx.doi.org/10.1007/978-1-4614-0586-3

Sharkey, J. D., You, S. y Schnoebelen, K. (2008). Relations among school assets, individual resilience, and student engagement for youth grouped by level of family functioning. Psychology in the Schools, 45(5), 402-418. http://dx.doi.org/ 10.1002/pits.20305

Simons-Morton, B. y Chen, R. (2009). Peer and parent influences on school engagement among early adolescents. Youth and Society, 41(1), 3-25. http://dx.doi.org/10.1177/0044118X09334861

Song, S. Y., Sikorski, J., Doll, B. y Sikorski, M. (2014). Enhancing classroom resilience with ClassMaps Consultation. En S. Prince-Embury, D. H. Saklofske, S. Prince-Embury, y D. H. Saklofske (Eds.), Resilience interventions for youth in diverse populations (pp. 203-215). New York, NY, US: Springer Science. http://dx.doi.org/10.1007/978-1-4939-0542-3_10

Sun, S., Pan, W. y Wang, L. (2010). A comprehensive review of effect size reporting and interpreting practices in academic journals in education and psychology. Journal of Educational Psychology, 102(4), 989-1004. http://dx.doi.org/10.1037/a0019507

Toland, J. y Carrigan, D. (2011). Educational psychology and resilience: New concept, new opportunities. School Psychology International, 32(1), 95-106. http://dx.doi.org/10.1177/0143034310397284

Ungar, M. y Liebenberg, L. (2013). Ethnocultural factors, resilience, and school engagement. School Psychology International, 34(5), 514-526. http://dx.doi.org/10.1177/0143034312472761

Veiga, F. H., García, F., Reeve, J., Wentzel, K. y Garcia, O. (2015). When adolescents with high self-concept lose their engagement in school. Revista de Psicodidáctica, 20(2), 305-320. http://dx.doi.org/10.1387/RevPsicodiact.12671

Veiga, F. H., Burden, R., Appleton, J., Céu, T. y Galvao, D. (2014). Student's engagement in school: conceptualization and relations with personal variables and academic performance. Revista de Psicología y Educación, 9(1), 29-47. 